\title{
Treatment of Acute Osteomyelitis with Once-Weekly Oritavancin: A Two-Year, Multicenter, Retrospective Study
}

\author{
Nicholas W. Van Hise ${ }^{1} \cdot$ Vishnu Chundi $^{1} \cdot$ Vishal Didwania $^{1} \cdot$ Michael Anderson ${ }^{1} \cdot$ David McKinsey $^{2} \cdot$ Ingrid Roig $^{3}$. \\ Akhilesh Sharma $^{4} \cdot$ Russell M. Petrak ${ }^{1}$
}

Published online: 25 June 2020

(c) The Author(s) 2020

\begin{abstract}
Background Acute osteomyelitis is typically caused by Gram-positive pathogens, commonly antibiotic-resistant Staphylococcus species. Standard antibiotic treatment is challenging due to required multiple daily doses, therapeutic drug monitoring, and parenteral administration for at least 4 weeks. Oritavancin is a long-acting lipoglycopeptide antibiotic approved as a single $1200 \mathrm{mg}$ dose for the treatment of adult patients with acute bacterial skin and skin structure infections.

Objective To characterize the real-world use, efficacy, and safety of oritavancin in adult patients with acute osteomyelitis. Methods This was a 2-year, multicenter, retrospective, descriptive study of patients treated for acute osteomyelitis with weekly doses of oritavancin. End of therapy evaluation (ETE) was defined as evaluation at 7-10 days after the last dose of oritavancin, and post-therapy assessment (PTE) was at 3 months and 6 months. At ETE and PTE, patients were interviewed via telephone for clinical outcomes, using a standard questionnaire. Electronic medical record review was also conducted. Results 134 patients were treated with oritavancin for acute osteomyelitis across 20 different Metro Infectious Disease Consultants infusion centers in six states. Of total positive cultures, $71.9 \%(92 / 128)$ were methicillin-resistant Staphylococcus aureus (MRSA) from deep wounds, bone, or joint culture; an additional nine (6.7\%) of 134 patients presented with concomitant MRSA bacteremia. Oritavancin was administered via intravenous catheter; patients received an initial treatment of $1200 \mathrm{mg}$ and then $800 \mathrm{mg}$ weekly thereafter for a total number of doses of four $(n=118)$ or five $(n=16) .118$ patients $(88.1 \%)$ of the baseline 134 patients achieved clinical success at the ETE timepoint. 130 patients were available for PTE at 3 months and 6 months. Overall, relapse or persistent infection was diagnosed in 13/134 (9.7\%) patients. Nine (6.7\%) of 134 patients were admitted to the hospital during the follow-up period but none for osteomyelitis. Adverse events were reported in five (3.7\%) patients including hypoglycemia-related symptoms (three patients), tachycardia (one patient), and tachycardia with chest pain (one patient). None of these patients were hospitalized due to adverse events, and all patients eventually finished their treatment regimens.

Conclusion This is the largest real-world clinical study of adult patients treated with oritavancin for acute osteomyelitis. Use of oritavancin for acute osteomyelitis infection resulted in a high rate of positive clinical outcomes and a low incidence of adverse events, thereby providing potential for a convenient, effective, and safe therapeutic option. Future prospective and comparative studies are needed to validate these findings.
\end{abstract}

Digital features To view digital features for this article go to https://doi.org/10.6084/m9.figshare.12221690.

Nicholas W. Van Hise

nvanhise@midcusa.com

1 Metro Infectious Disease Consultants, 901 McClintock Dr., Ste. 202, Burr Ridge, IL, USA

2 Metro Infectious Disease Consultants, Kansas City, MO, USA

3 Metro Infectious Disease Consultants, Huntsville, AL, USA

4 Metro Infectious Disease Consultants, Avondale, AZ, USA

\section{Introduction}

Acute osteomyelitis in adults is a common therapeutic dilemma, yet treatment algorithms and institutional guidelines are difficult to construct due to the heterogeneous nature of osteomyelitis, lack of well-designed intervention studies, involvement of joint prostheses versus native joints, and large variations in patient factors and co-morbidities. In addition, the lack of US Food and Drug Administration (FDA) guidance for clinical trial methodology and efficacy evaluation standards precludes consistent drug development 


\section{Key Points}

Acute osteomyelitis is clinically challenging to treat as it is commonly caused by Gram-positive pathogens, particularly resistant Staphylococcus, and antibiotic treatment requires multiple daily doses, therapeutic monitoring, and parenteral administration for multiple weeks.

In real-world clinical use, oritavancin doses of 1200 $\mathrm{mg}$ and then $800 \mathrm{mg}$ once per week for 4-5 weeks for treatment of acute osteomyelitis showed positive clinical outcomes-high rate of clinical success and low rate of adverse events.

for this indication. In practice, debridement of infected tissue and surgical resection, sometimes with removal or revision of prostheses, are followed by an extended course of parenteral or oral antibiotics, commonly 4-6 weeks in duration. Antibiotic selection, route of administration, and duration of therapy are guided by clinician assessment, clinical experience, and patient preference due to lifestyle factors (i.e., lack of transportation, adherence concerns, etc.) [1].

Oritavancin is a novel lipoglycopeptide antibiotic with a long terminal half-life of $245 \mathrm{~h}$ and in vitro activity against a broad spectrum of Gram-positive organisms including methicillin-resistant Staphylococcus aureus (MRSA), methicillin-sensitive Staphylococcus aureus (MSSA), various Streptococcus spp., and Enterococcus faecalis (vancomycin-susceptible isolates only), and has demonstrated potent in vitro activity against vancomycin-resistant enterococci such as E. faecium [2-4]. This was a multicenter, retrospective, observational study describing the utilization, clinical outcomes, and adverse events (AEs) in patients treated with oritavancin in a clinical setting for acute osteomyelitis.

\section{Methods}

Consecutive patients who received oritavancin $1200 \mathrm{mg}$ followed by $800 \mathrm{mg}$ once weekly for acute osteomyelitis were included in this retrospective review. Patients who received any other dosing regimen were excluded. Diagnosis of acute osteomyelitis and antibiotic treatment decisions were determined by board-certified infectious disease physicians at multiple sites throughout the USA. All patients received a total treatment duration of 4 or 5 weeks. All oritavancin infusions were administered over $3 \mathrm{~h}$. Clinical outcomes were assessed at the completion of the entire oritavancin course of therapy 7-10 days after the end of the last dose (ETE) and at 3 months and 6 months post-treatment (PTE) using both retrospective electronic medical records assessment and patient telephone interviews conducted by an infectious diseasetrained pharmacist using a Western Institutional Review Board (WIRB)-approved questionnaire and data collection form. Clinical outcome determinations at ETE were based on the following:

1. Clinical success based on resolution of symptoms or improvement in symptoms and no further need for treatment; or,

2. Clinical failure based on lack of improvement in symptoms and need for use of additional Gram-positive antibiotics for osteomyelitis, admission to hospital for osteomyelitis, or loss to follow up.

Clinical success and failure at PTE were determined during the 3- and 6-month follow-up timeframe.

Safety data, including discontinuation of oritavancin due to safety concerns, infusion events, and other adverse events as noted by patient telephone interview at 7-10 days, 3 months, and 6 months post-oritavancin were reported. The retrospective study was approved for analysis and publication by WIRB and patient consent was waived.

\section{Results}

One-hundred and thirty-four adult patients were treated with oritavancin for acute osteomyelitis as diagnosed by clinical assessment, baseline MRI, microbiology, and inflammatory markers between 1 January 2016 and 31 December 2018. Baseline characteristics are listed in Table 1.

Patients received infusions at 20 infusion centers in six states under the care of 37 infectious diseases specialists. The selection of oritavancin as the therapeutic agent of choice was based on patient preference or transportation challenges in $89.6 \%$ of cases.

Microbiologic analysis recovered 121 (90.3\%) positive cultures from debridement of bone or joint and seven $(5.2 \%)$ from curettage of wounds. Culture results were positive in 119 (88.8\%) patients with several cultures confirming a polymicrobial Gram-positive infection.

MRSA was the most common pathogen isolated (92/128; $71.9 \%)$ and $80.5 \%$ (74/92) were monomicrobial. Baseline bacteremia was identified in nine $(6.7 \%)$ patients; all patients had a retained indwelling intravenous catheter placed during hospitalization. Repeat blood cultures were performed in all patients with baseline bacteremia and revealed clearance of pathogens; see Table 2 for microbiology.

Clinical outcomes are shown in Table 3. Clinical outcomes were reported at the ETE timepoint for 134 patients and at the PTE for 130 patients. Clinical success was observed in $118(88.1 \%)$ of 134 patients at ETE; all evaluable patients (excluding patients lost to follow-up) with 
Table 1 Baseline demographics in 134 osteomyelitis cases

\begin{tabular}{|c|c|}
\hline & $N(\%)$ \\
\hline Male & $66(49.3)$ \\
\hline Female & $68(50.7)$ \\
\hline Age, years, mean (range) & 60 (19-97) \\
\hline \multicolumn{2}{|l|}{ Age group, years } \\
\hline$<30$ & $7(5.2)$ \\
\hline $31-49$ & $28(20.9)$ \\
\hline $50-65$ & $54(40.3)$ \\
\hline$\geq 66$ & 45 (33.6) \\
\hline Weight, $\mathrm{kg}$, mean (range), SD & 78 (38-164), 23.8 \\
\hline BMI $\left(\mathrm{kg} / \mathrm{m}^{2}\right)$, mean, (range), SD & $27(15.8-48.4), 6.7$ \\
\hline Baseline MRI & $134(100)$ \\
\hline Infection confirmed on MRI & $128(95.5)$ \\
\hline Baseline CRP & $134(100)$ \\
\hline Baseline ESR & $132(98.5)$ \\
\hline Previous antibiotic therapy & $18(13.4)$ \\
\hline Baseline bacteremia (MRSA) & $9(6.7)$ \\
\hline Debridement of bone or joint & $121(90.3)$ \\
\hline Positive wound, bone, joint culture & $119(88.8)$ \\
\hline Culture positive (wound, joint, deep wound) MRSA & $\begin{array}{l}92 / 128 \text { with cultures } \\
\text { obtained (71.9) }\end{array}$ \\
\hline Prosthetic source & $24(17.9)$ \\
\hline \multicolumn{2}{|l|}{ Anatomic location of infection } \\
\hline Lower extremity source & $74(55.2)$ \\
\hline Upper extremity source & $25(18.7)$ \\
\hline Pelvic source & $18(13.4)$ \\
\hline Head and shoulder source & $9(6.7)$ \\
\hline Vertebral source & $8(6)$ \\
\hline \multicolumn{2}{|l|}{ Renal function at baseline } \\
\hline $\mathrm{CrCl}>61 \mathrm{~mL} / \mathrm{min}$ & $89(66.4)$ \\
\hline $\mathrm{CrCl} 31-60 \mathrm{~mL} / \mathrm{min}$ & $12(9)$ \\
\hline $\mathrm{CrCl} 15-30 \mathrm{~mL} / \mathrm{min}$ & $8(6)$ \\
\hline ESRD & $7(5.2)$ \\
\hline \multicolumn{2}{|l|}{ Co-morbidities } \\
\hline$\geq 3$ co-morbidities & $57(42.5)$ \\
\hline Hyperlipidemia & $59(44)$ \\
\hline Diabetes & $51(38.1)$ \\
\hline Peripheral vascular disease & $36(26.9)$ \\
\hline COPD & $33(24.6)$ \\
\hline History of MI & $25(18.7)$ \\
\hline Congestive heart failure & $25(18.7)$ \\
\hline Malignancy & $20(14.9)$ \\
\hline Peptic ulcer disease & $13(9.7)$ \\
\hline Current chemotherapy/immunotherapy & $13(9.7)$ \\
\hline \multicolumn{2}{|l|}{ Miscellaneous } \\
\hline IVDU (past or present) & $6(4.5)$ \\
\hline HIV positive & $5(3.7)$ \\
\hline Liver disease & $3(2.2)$ \\
\hline Chronic steroids ( $\geq 20 \mathrm{mg} /$ day prednisone) & $1(0.75)$ \\
\hline Chronic kidney insufficiency & $33(24.6)$ \\
\hline $\begin{array}{l}\text { Patient preference or transportation issues as reason for } \\
\text { use of oritavancin }\end{array}$ & $120(89.6)$ \\
\hline
\end{tabular}

$B M I$ body mass index, $\mathrm{CrCl}$ creatinine clearance, $\mathrm{CRP}$ C-reactive protein, ESR erythrocyte sedimentation rate, ESRD end-stage renal disease, COPD chronic obstructive pulmonary disease, IVDU intra-
Table 1 (continued)

venous drug user, $M I$ myocardial infarction, $M R I$ magnetic resonance imaging, MRSA methicillin-resistant Staphylococcus aureus, MSSA methicillin-sensitive Staphylococcus aureus, $S D$ standard deviation

clinical success at ETE carried forward to PTE showed a clinical success rate of 91.2\% (104/114). Overall, of 130 patients evaluable at both ETE and PTE timepoints, clinical success was seen in $80.0 \%$ (104/130). Only four patients were lost to follow-up during the 6-month evaluation period. During the PTE follow-up time frame, there were nine $(6.7 \%)$ patients admitted to the hospital but none for osteomyelitis, so these were not included as clinical failures. Thirteen patients $(9.7 \%)$ were deemed to have a persistent or relapsing infection (nine patients at ETE and four at PTE) and thus were classified as clinical failures. In another six patients, the need for additional antibiotic therapy post-oritavancin treatment was counted as a clinical failure.

Post-treatment imaging was not routinely performed, and no additional culture data were collected; however, CRP (C-reactive protein) and ESR (erythrocyte sedimentation rate) values were decreased by at least $50 \%$ in $134(100 \%)$ and 123 (93.2\%) of patients, respectively, at ETE.

AEs were evaluated during the entire 6-month period and occurred in five patients. All AEs occurred during the oritavancin infusion upon infusion of either the second or the third dose. Three patients experienced hypoglycemia-related symptoms. Two of the five patients had tachycardia during the infusion, of whom one experienced chest pain. Oritavancin infusion was discontinued for both patients, diphenhydramine was administered, and symptoms resolved. Both

Table 2 Baseline microbiology

\begin{tabular}{|c|c|}
\hline Culture and pathogen by unique patient & $n / N(\%)$ \\
\hline Positive cultures with $\geq 1$ GP result & $119 / 134(88.8)$ \\
\hline \multicolumn{2}{|l|}{ Staphylococcus aureus, monomicrobial } \\
\hline MRSA & 92 \\
\hline MSSA & 25 \\
\hline \multicolumn{2}{|l|}{ S. aureus, mixed } \\
\hline MRSA $+\geq 1$ other GP pathogen ${ }^{a}$ & 16 \\
\hline MSSA $+\geq 1$ other GP pathogen ${ }^{\mathrm{a}}$ & 10 \\
\hline Vancomycin-resistant enterococci ${ }^{\mathrm{b}}$ & 7 \\
\hline Vancomycin-intermediate $S$. aureus $^{\mathrm{b}}$ & 2 \\
\hline VRE with daptomycin $\mathrm{MIC} \geq 4 \mathrm{mg} / \mathrm{L}$ & 2 \\
\hline
\end{tabular}

MRSA methicillin-resistant Staphylococcus aureus, MSSA methicillin-sensitive Staphylococcus aureus, GP Gram-positive

${ }^{a}$ Includes Streptococcus pyogenes, group B streptococci, coagulasenegative staphylococci, and Enterococcus spp

${ }^{\mathrm{b}}$ Identified in polymicrobial Gram-positive infections, usually with MRSA or MSSA 
Table 3 Clinical outcomes

\begin{tabular}{lll}
\hline & No. of patients, $n(\%)$ & Clinical cure, $n(\%)$ \\
\hline Clinical success ETE & 134 & $118(88.1)$ \\
Clinical success PTE & 130 & $104(80.0)$ \\
Subgroups evaluated at ETE & & \\
Four-dose regimen & $118(88.1)$ & $107(90.7)$ \\
Five-dose regimen & $16(11.9)$ & $11(68.8)$ \\
MRI-proven infection & $128(95.5)$ & $113(90.4)$ \\
Diabetes & $51(38.1)$ & $43(84.3)$ \\
Prosthetic device & $24(17.9)$ & $20(88.3)$ \\
Heart failure & $25(18.7)$ & $21(84)$ \\
Previous antibiotic therapy & $18(13.4)$ & $14(77.8)$ \\
Malignancy on immunosuppression & $12(9)$ & $11(91.7)$ \\
\hline
\end{tabular}

ETE end of the last dose, MRI magnetic resonance imaging, PTE post-treatment

patients were evaluated in the emergency room, but were not admitted to the hospital. Both patients eventually finished their course of oritavancin therapy. All patients finished treatment regimens.

\section{Discussion}

In this study, use of oritavancin for Gram-positive osteomyelitis resulted in positive clinical outcomes, providing further evidence that oritavancin may be a convenient, effective, and safe therapeutic option for treatment of adult patients with osteomyelitis. These results are similar to previously published real-world evidence from several case reports and studies describing the use of single- and multiple-dose regimens with oritavancin for a variety of complex infections, including osteomyelitis.

Indeed, the Clinical and Historic Registry and Orbactiv Medical Evaluation (CHROME) study (2014-2017) reported clinical success was observed in 93.8\% (30/32) of patients treated with multiple doses of oritavancin separated by 14 days or less for a variety of infections [4]. Eight of these patients received multiple doses (two to ten) of oritavancin for osteomyelitis, and seven $(87.5 \%)$ had a positive clinical response. Additionally, ten patients completed therapy for Gram-positive osteomyelitis with a single dose of oritavancin and demonstrated a clinical success of $90.0 \%$. Oritavancin was safe and well tolerated in the CHROME study; 29 (6.6\%) patients experienced at least one AE compared to the five (3.7\%) in the present study [4].

Schulz and colleagues reported 17 patients who received multiple doses of oritavancin (two to 18 doses) for the treatment of complicated Gram-positive infections including four patients with osteomyelitis [5]. All osteomyelitis patients achieved clinical success with oritavancin, and four patients experienced AEs during the oritavancin infusion that resolved after discontinuation [5]. Another case series by Chastain et al. included nine patients who were treated for lower-extremity chronic osteomyelitis with at least two doses of oritavancin; they observed clinical cure at 6 months' follow-up in all patients without a report of an AE [6]. The results of both case series concur with the present study results.

Several single patient case reports are available in the literature describing use of multiple doses of oritavancin in the treatment of bone or joint infection. Delaportes et al. [7] treated a patient with six once-weekly doses of oritavancin for MSSA osteomyelitis; Antony et al. [8] treated two patients with prosthetic joint infections who had single-stage revisions, antibiotic spacer, and two to four doses of oritavancin infused 10 days apart; Foster et al. [9] published a case of a patient who received oritavancin for femoral osteomyelitis due to VRE and was treated with six once-weekly doses. Although these data reflect individual cases or small case series, the data illustrate the interest in use of a longacting antibiotic, such as oritavancin, for complex clinical situations such as osteomyelitis.

Administration of oritavancin does not require patients to be hospitalized and treatment can occur in outpatient settings. Additionally, oritavancin dosing - whether as a single dose or once per week for multiple-dose regimens, offers logistical advantages over traditional therapies that require parenteral administration for at least 4 weeks.

Limitations to the present study include the retrospective, observational, non-comparative methodology and unknown influence of prior antibiotic therapy. A major strength of this study is the large number of patients treated with a mostly consistent dosing regimen evaluated across 20 US-based facilities in several states. Another strength is the prevalence of patients with multiple co-morbidities, which has been reported as a risk factor for failure of antibiotic therapies regardless of infection type. Data collection and analysis 
were carried out by a single well-trained infectious diseases pharmacist. Since there are no randomized, well-controlled studies evaluating the safety and efficacy of multiple doses of oritavancin for the treatment of osteomyelitis, these results provide a significant contribution to the literature to be considered when making treatment decisions.

\section{Conclusion}

In this retrospective study of real-world data, the use of oritavancin for the treatment of adult patients with Grampositive acute osteomyelitis resulted in successful clinical outcomes. This provides further evidence that oritavancin may be an effective and safe therapeutic option.

Acknowledgements The authors wish to thank the physicians, pharmacists, and nursing staff of Metro Infectious Disease Consultants for their support with this project.

Author contributions All authors had a role in study design and in conceiving and writing the manuscript. According to the guidelines of the International Committee of Medical Journal Editors (ICMJE, www. icmje.org) all authors met the criteria for authorship and no deserving authors have been omitted.

Data Availability All data generated or analyzed during this study are included in this published article.

\section{Compliance with Ethical Standards}

Funding No sources of funding were used to assist in the preparation of this article. This article is part of a supplement wholly funded by Melinta Therapeutics.

Conflict of interest NVH has received speaker fees for Vabomere, which is owned by Melinta Therapeutics. VC, VD, MA, DM, IR, AS, and RP have no disclosures.

Ethics approval This retrospective study was approved for analysis and publication by the Western Institutional Review Board and has been performed in accordance with the ethical standards of the Declaration of Helsinki. Informed consent was waived as this study was retrospective.

Open Access This article is licensed under a Creative Commons Attribution-NonCommercial 4.0 International License, which permits any non-commercial use, sharing, adaptation, distribution and reproduction in any medium or format, as long as you give appropriate credit to the original author(s) and the source, provide a link to the Creative Commons licence, and indicate if changes were made. The images or other third party material in this article are included in the article's Creative Commons licence, unless indicated otherwise in a credit line to the material. If material is not included in the article's Creative Commons licence and your intended use is not permitted by statutory regulation or exceeds the permitted use, you will need to obtain permission directly from the copyright holder.To view a copy of this licence, visit http://creativecommons.org/licenses/by-nc/4.0/.

\section{References}

1. Cortes-Penfield NW, Kulkarni PA. The history of antibiotic treatment of osteomyelitis. Open Forum Infect Dis. 2019;6(5):ofz181.

2. Yan Q, Karau MJ, Patel R. In vitro activity of oritavancin against planktonic and biofilm states of vancomycin-susceptible and vancomycin-resistant enterococci. Diagn Microbiol Infect Dis. 2018;91(4):348-50.

3. Belley A, Arhin FF, Moeck G. Evaluation of oritavancin dosing strategies against vancomycin-resistant Enterococcus faecium isolates with or without reduced susceptibility to daptomycin in an in vitro pharmacokinetic/pharmacodynamic model. Antimicrob Agents Chemother. 2018;62(1):e01873-17.

4. Redell M, Moeck G, Lucasti C, Durso S, Kennedy C, Fusaro K, et al. A real-world patient registry for oritavancin demonstrates efficacy and safety consistent with the phase 3 SOLO program. Open Forum Infect Dis. 2018;5(6):ofy051.

5. Schulz LT, Dworkin E, Dela-Pena J, Rose WE. Multiple-dose oritavancin evaluation in a retrospective cohort of patients with complicated infections. Pharmacotherapy. 2018;38(1):152-9.

6. Chastain DB, Davis A. Treatment of chronic osteomyelitis with multidose oritavancin: a case series and literature review. Int J Antimicrob Agents. 2019;53(4):429-34.

7. Delaportas DJ, Estrada SJ, Darmelio M. Successful treatment of methicillin susceptible Staphylococcus aureus osteomyelitis with oritavancin. Pharmacotherapy. 2017;37(8):e90-e9292.

8. Antony SJ, Cooper LG. Use of oritavancin (novel new lipoglycopeptide) in the treatment of prosthetic joint infections (PJI): a possible alternative novel approach to a difficult problem. Infect Disord Drug Targets. 2017;17(2):77-80.

9. Foster R, Philavong K, Weissman S, Tang X, Bookstaver B. Oritavancin for treatment of daptomycin nonsusceptible vancomycin-resistant enterococci osteomyelitis. Infect Dis Clin Pract. 2017;26(2):1. 